\title{
Mobile ECG Scanner Using Smartphone
}

\author{
Sami M. Halawani and Sarudin Kari \\ Faculty of Computing and Information Technology \\ King Abdulaziz University at Rabigh \\ halawani@kau.edu.sa_sarudin.kari@gmail.com
}

\begin{abstract}
ECG (electrocardiogram) is a test to measure the electrical activity of the heart by applying electrical impulses. The information obtained from an ECG can be analyzed to discover different types of heart diseases. It is useful to monitor a patient's respond to a specific treatment. Some patients may need to move around while still being monitored by an ECG. Hence, it is important to have a mobile ECG solution. Building a stable and reliable mobile ECG solution needs huge investment especially if all main components are built from scratch. This research proposes to build the mobile ECG solution using available main components from the shelf. Bluetooth is used instead of USB in order to provide future flexibility to add more electrodes. In order to reduce the size, development cost, and time, Smartphone is used as the host PC. Present Smartphone have sufficient resources to provide the necessary data logging, HMI and remote monitoring.
\end{abstract}

Keywords: ECG, Smartphone, Bluetooth, HMI

\section{Introduction}

In some countries, heart diseases have become more frequent and fatal to the population. However, it is costly to monitor the heart of every potential patient without reducing the size, and power consumption of the monitoring equipment. Reducing the size of the monitoring system provides the mobility which is necessary for many potential patients. ECG (electrocardiogram) is a test that is widely used to monitor a patient's heart condition. Due to its bulky size, a patient is tied to a bed or chair so that the patient can be monitored continuously. As a result, the 
patient has limited mobility and cannot do anything else. While having a heart disease, it does not mean the patient is not healthy enough to do other activity. Thus, it is important to have an ECG system which is small size and able to be moved around.

Although building an ECG system is relatively easy, to make the system stable and reliable is more difficult than it seems. Since an ECG system is very sensitive to human safety, it is important that the system being deployed is very stable, reliable and safe to the user. A system being developed from scratch need extensive test to determine the stability and reliability of a system. Ingenuity technique may be needed to filter any noise at the lower $\operatorname{cost}^{[3]}$. In order to avoid the time and cost for extensive test, it is proposed to use existing components available on the shelf. Nowadays, Smartphone are getting more powerful, less power consumption and many added features ${ }^{[9]}$ which can be optimized to build a complete ECG solution. Some of the extensive works on ECG was mention $\left.\left.\mathrm{in}^{[1}\right]^{[}[2]^{[3]}\right]$. The issues of mobility were discussed in $\left.^{[4], 6[6][7}\right]$. Smartphone provides the option to easily be connected to a server for remote monitoring. However, building a Smartphone application is not as easy as building an application for a PC. This research is going to focus on building an ECG solution based on Android Smartphone for various reasons.

This paper was written in 6 sections. After introduction in section 1 , the review on each main component is presented in section 2 . The system architecture is shown in Section 3. The development work is shown in Section 4. The result and conclusion is available in Section 5 and 6 respectively.

\section{ECG and Smartphones}

The proposed solution was developed as a SCADA (Supervisor Control and Data Acquisition) system in mind. There are four main components: sensor and interface, controller, host PC with server, and communication.

\subsection{ECG Sensors and Interface}

ECG (electrocardiogram) is a test that is widely used to monitor a patient's heart condition. It is a non-invasive diagnostic tool to measure the electrical activity of the heart. The heart is an internal organ that beats 
in rhythm to pump the blood through the body. The study of this electrical signal can help in determining many abnormalities related to the heart's function.

The ECG was first put to clinical use in 1913 with Einthoven's invention of the string galvanometer. Einthoven's recording is known as the "three lead" ECG, with measurements taken from three points on the body. The difference between potential readings from point 1 and point 2 is what is used to produce the output ECG trace. The point 3 connection is needed to establish a common ground for the body and the recording device.

For a mobile system, the information obtained from an ECG must be able to be analyzed on the fly to discover different types of heart disease. The mobile system can also be used as a data logger for future analysis and treatment. The data logging is useful for seeing how well the patient is responding to a treatment. In mobile unit, the heart beating is stored as a series of database which later can be uploaded into a server or printed for further analysis ${ }^{[8]}$. Using mobile unit, the patient can be monitored 24 hours per day and 7 days per week either while doing hard and stressing activities or relaxing at home. The following figure is a sample of how an ECG looks like.

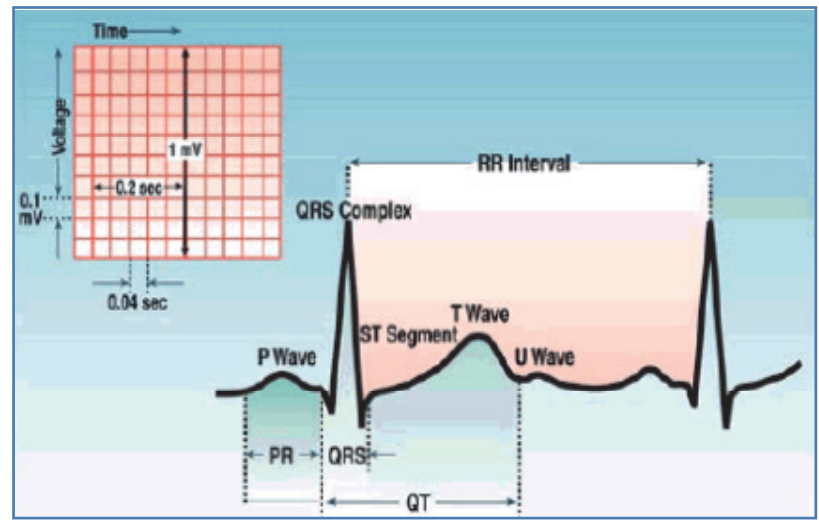

Fig. 1. ECG Reading of a Heart.

The heart anamolies or diseases are detected by analyzing the time between different points of $\mathrm{P}, \mathrm{Q}, \mathrm{R}, \mathrm{S}, \mathrm{T}$ and $\mathrm{U}$, and the variations in these times between successive beats. The $\mathrm{P}$ wave represents atrial activation. The PR interval is the time from onset of atrial activation to onset of ventricular activation. The QRS complex represents ventricular 
activation; the QRS duration is the duration of ventricular activation. The ST-T wave represents ventricular repolarization. The QT interval is the duration of ventricular activation and recovery. The $U$ wave probably represents "after depolarizations" in the ventricles.

The simple block diagram of the sensors, interface and controller is shown in Fig. 4. The details of the proposed system are shown in Fig. 2. A cheap microcontroller such as Microchip PIC with integrated analog to digital converter can be used.

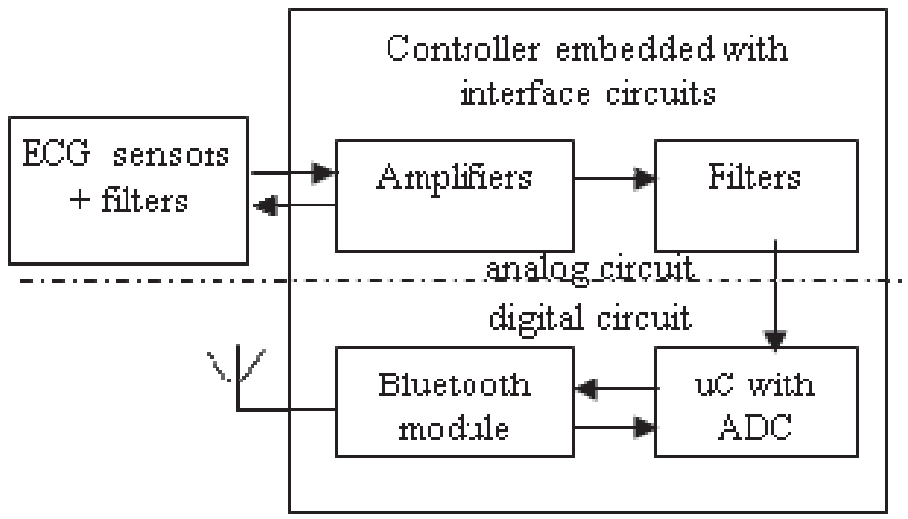

Fig. 2. ECG Controller Architecture.

Typical ECG sensors' output is between 0.5 to $2 \mathrm{mV}$. To ensure low cost ECG solution, the ECG sensors must be cheap yet reliable and easy to be used or stored. The sensors or electrodes are normally disposed to increase safety to other patients. In order to reduce the ECG solution cost in the long run, reusable electrodes must be made from cheap material such as metal plates or strips with large areas to minimize electrode resistance. An example of a cheap and reusable electrode is shown in Fig. 3. The sensors must be built from easily available resource such as copper coins.

Hence, a pre-amplifier is needed before the actual amplifier is applied. Once amplified, filters are applied to get rid any noise due to power supply interference, and surrounding noise. The output of the filters is fed into the analog to digital converter which is available inside the microcontroller. In microcontroller, the ECG is converted into digital signal by sampling technique. Two channels must be provided for a single electrode ECG to read the point 1 and point 2 voltages. 


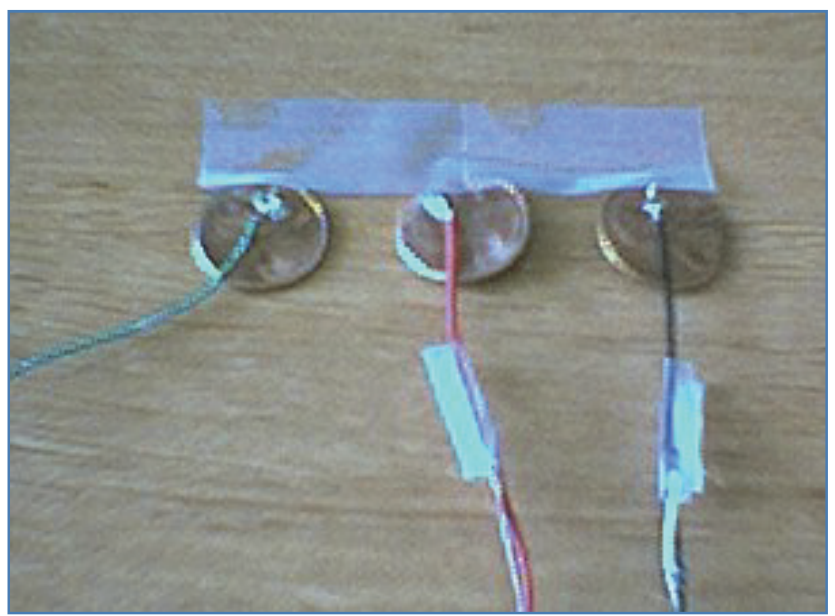

Fig. 3. Simple and Disposable Electrode.

\subsection{ADC and Microcontroller}

To reduce cost, interface circuits, filters and microcontroller are built in a single PCB. The microcontroller must have an integrated analog to digital converter, program memory, and RAM to save more space and power consumption. The main task of the microcontroller is just to digitize the electrode reading and send the digitized data to the Smartphone. Thus, the microcontroller needs a very minimum processing power.

However, microcontroller can be used to build digital filter such as in ${ }^{[3]}$. Thus, the ECG raw data can be improved further by applying various types of digital filters. This also means the analog filters can be removed to save space and power consumption. Digital filters are more stable and maintain accuracy at different temperatures. As a result, the ECG graph can be produced at better accuracy.

The microcontroller also can be used to interface with Bluetooth module. This is important so that the connection between the controller and the Smartphone is wireless. Reducing the wire connection gives more freedom to the patient.

\subsection{Communication}

There are two types of communication in the ECG solution: local communication and remote communication. The local communication is the connection between the controller and sensors, and connection 
between the controller and the Smartphone. It is better to use shielded wire between the ECG sensors and controller so that the noise can be reduced to the minimum. The shielded wire must be also as short distance as possible. The short distance is possible since the microcontroller board can be put at the distance from the Smartphone due to the Bluetooth connectivity.

Having wireless communication added safety to the user. Now, the power source between the two modules is totally isolated. The controller is using very low power compared to the Smartphone. In addition, total power isolation increase reliability due to less noise interaction between the two modules.

A Smartphone already has many multi mode of communication such as SMS, GPRS, 3G, 4G, and WiFi. The multi mode communication gives the option to transfer data at maximum speed if the highest bandwidth is available. It also gives the option that even in the worst signal strength, there are still means to transfer the data to the server.

\subsection{Smartphone as Host PC}

When this research started, Nokia Smartphone based on Symbian OS is the majority of Smartphone being sold annually. Looking at the sales trend, Smartphone based on Android OS was gaining a lot of sales traction. Thus, Smartphone based on Android or Android Smartphone will be the majority of the most sold Smartphone ${ }^{[9]}$. This is an important factor to be considered because of two reasons. First, the more sales a type of Smartphone, the less cost it takes to produce. The cost saving can be passed to the consumer by selling the product at lower cost. Thus, the ECG solution can be sold at a very attractive price. Second, unlike a PC application development, a Smartphone application is developed for a specific Smartphone OS. This is a very important factor considering that if in the future patients may already have their own Smartphone, they only need to buy the integrated ECG controller and the application program.

However, Android Smartphone is not only produced by a single company. Many companies use Android OS and customized the OS for their specific needs or Smartphone features. Thus, there are compatibility issues in developing applications for Smartphone. Even between Smartphone brands from the same company, there are possibilities of 
incompatibility due to different Android versions, Smartphone features, and resources available. Due to this compatibility issues, $\mathrm{C}++$ language is not suitable if the application to be built once and used by any Android Smartphone. The best alternative programming language for compatibility purpose is JAVA language.

\subsection{Server}

There are two main reasons for the Smartphone to exchange data with a server. First, the Smartphone does not have sufficient processing power to cater for all heart disease analysis, identification and recognition. Thus, by sending critical data to the server, a more thorough analysis can be done at real time $\left.\left.{ }^{[1}\right]^{[8}\right]$. The result can be sent back to the Smartphone to notify the patient. Second, data available is needed for secured data logging in future references. Smartphone have limited data storage or the patient may incidentally damage the data. Thus, by storing the data in the server, the data is always available for future use.

The server is where the actual data logging should be built. The data logging in the Smartphone is used temporarily or for limited time data only. However, it is not the intention of this research to build a complete and optimize data logging at the server. For prove of concept purpose, a simple data logging is built in the server using Microsoft Access database to store the daily data.

\section{Architecture Development}

\subsection{Architecture}

This research proposed to develop a complete solution to monitor heart disease at two levels: At the patient proximity and at a remote location. The simplified architecture is shown in Fig. 4. The controller and Smartphone are attached to the patient all the time. Both act as the first level of monitoring where only a specific or two type of heart diseases are monitored. Similar data is being transmitted to the server, other possible heart diseases are monitored at the server. This scheme is chosen because no matter how advance a Smartphone, it still has limited resources and processing power. In addition, patient still need to use the Smartphone for other purpose especially for emergency call. 


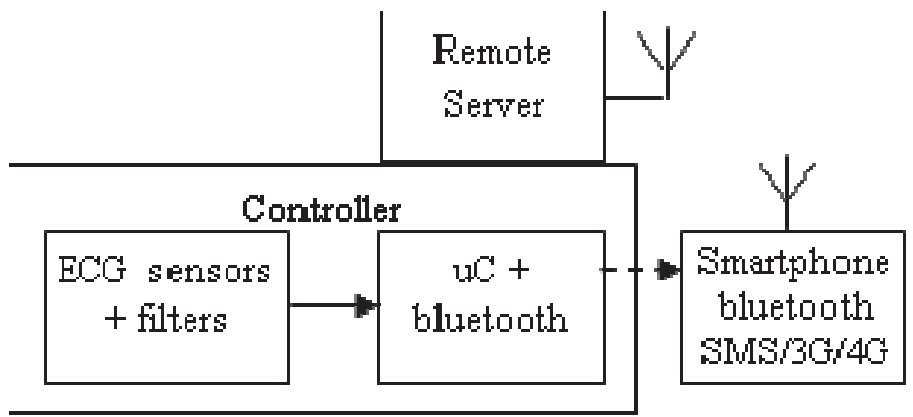

Fig. 4. Propose Mobile ECG on Smartphone.

Since the Smartphone is free from wire connection to the controller, the patient can freely use the Smartphone for other functions. In addition, the patient is monitored 24 hours per day and 7 days per week both by the Smartphone and also the server. If there is anything wrong with the Smartphone, the operator of the server can quickly send an alert to health personnel for immediate action.

\subsection{Development Issues}

There are many sources of noise being introduced into the system mainly at the controller. Some of the noises are solved when Bluetooth communication was chosen. Another solution is to ensure to use special cream between the electrode and the patient skin. Wiring sensors directly to Smartphone will make the Smartphone inflexible to be moved around especially calling and using other applications. Wiring the sensors to USB directly also increase power consumption and locking the USB for specific application. This is another reason Bluetooth communication was chosen.

\subsection{Smartphone Application}

The Smartphone acts as a miniature Host PC. The application program was built to have Human Machine Interface (HMI), database and communication. There are two kinds of communication involve: Local and remote. The local communication is between the Smartphone and the controller. The remote communication is between the Smartphone and remote server. The database is used to store the daily data of the raw heart beat and after analyzed heart beat data. For simplicity, the database is stored in text files and the filename is based on the date. These files will be sent to the server via wireless communication. 
The HMI was built to assist the user navigate through the application. It was made to be as simple as possible in order not to confuse the user. There is no graph display but more on menu selection and indication. All three activities were written in JAVA programming language.

\subsection{JAVA Platform}

After considering the problems which may arise in Section 2.4, the Smartphone application was developed using JAVA language. Although JAVA language produces slower application compared to $\mathrm{C}++$, the performance speed is sufficient for this application. The most important thing is JAVA language ensures program compatibility between Smartphone models for the same brand, and between Smartphone brands. In addition, there are extensive existing library for Smartphone application development can be used which speed up the development process.

\subsection{Controller Platform}

Microchip PIC 18F458 was used as the controller. Development was done using $\mathrm{C}$ language. Microchip PIC was chosen due to its variety and compatibility between the families of PIC. PIC families also have sufficient processing resources to do data acquisition, data filtering, and local communication. It is the most shipped microcontroller in the world since 2006 replacing Motorola (Freescale) microcontrollers.

The rate of data acquisition is based on Nyquist theorem which is two times the maximum frequency of the signal under study. Assuming a heart beat is between $20 \mathrm{~Hz}$ to $200 \mathrm{~Hz}$, the sampling frequency is between 40 to $400 \mathrm{~Hz}$. However, to avoid aliasing, the data is oversampling at 4 times the original data. Thus the sampling frequency is 80 to $800 \mathrm{~Hz}$. This is sufficiently low rate compared to Microchip PIC analog input sampling at around $150 \mathrm{KHz}$ for $40 \mathrm{MHz}$ oscillator.

\section{Result}

The proposed solution was successfully developed as intended in Fig. 2. Further work is needed to complete the solution as in Fig. 4. Although a simpler version was successfully transmitted data to a server 
(PC), a more thorough research is needed to increase the performance as intended.

The local interface and controller was successfully developed using microcontroller PIC18F458 or similar devices in the PIC family. The Smartphone was successfully tested using HTC Android wildfire. The HMI has a sufficient performance to display the data logging.

\section{Conclusion}

It is possible to develop a cheap mobile ECG solution using available components on the shelf as shown in this project. The propose solution was developed as an alternative to available fixed location ECG machine used in hospitals. However, there are several other issues must be explored such as digital filters using cheap microcontroller, secured data communication between the devices, and data compression. It is also possible that this solution is more efficient to be used for only a limited number of heart diseases while the rest are monitored at the remote server.

\section{Acknowledgement}

We would like to thank the Deanship of Scientific Research, King Abdulaziz University for providing us with the opportunity and assistance to conduct this research. This work was carried out under the contract number 430/007-27.

\section{References}

[1] Liang, H., ECG Feature Elements Identification For Cardiologist Expert Diagnosis, Proceedings of the 2005 IEEE Engineering in Medicine and Biology 27th Annual Conference, Shanghai, China, 2005: 3845-3848.

[2] Mukhopadhyay, S.K., Mitra, M. and Mitra, S., An ECG Data Compression Method via RPeak Detection and ASCII Character Encoding, International Conference on Computer, Communication and Electrical Technology (ICCCET), 2011 Tamilnadu: 136 - 141

[3] Zhang, W. and Ge, L., A Method for Reduction of Noise in the ECG. The 2nd International Conference on Bioinformatics and Biomedical Engineering, 2008, ICBBE 2008, Shanghai: $2119-2122$.

[4] Lin, C., Luo, D. and Wu, S., File Transmission via socket from Smartphone to PC Based on Symbian OS. International Conference on Wireless Networks and Information Systems 2009, Shanghai, China: $81-84$.

[5] Zhang, L., Liu, Y. and Guo, W., Research on Diversified Designing Methods and User Evaluation of Smartphone Interface. International Symposium on Computational Intelligence and Design (ISCID), 2010, 2: 10-13. 
[6] Friedman, R., Kogan, A. and Krivolapov, Y., On Power and Throughput Tradeoffs of WiFi and Bluetooth in Smartphones. Proceedings IEEE INFOCOM, 2011: 900 - 908.

[7] Zhang, L. and Liu, Y., On Methods of Designing Smartphone Interface. IEEE International Conference on Software Engineering and Service Sciences (ICSESS), 2010: 584-587.

[8] Yuan, B. and Herbert, J., Web-based Real-time Remote Monitoring for Pervasive Healthcare, IEEE International Conference on Pervasive Computing and Communications Workshops (PERCOM Workshops), 2011: 625 - 629

[9] Latif, L., Most phones shipped in 2015 will be Smartphones, Retrieved: 08-28-2011, from http://www.theinquirer.net/ inquirer/news/2104874/phones-shipped-2015-Smartphones. 


\section{جهاز تخطيط القلب المتحرك باستخدام الهاتق الذكي}

\section{سامي حلواني، وسارودين كاري}

كلية الحاسبات وتقنية المعلومات، فرع جامعة الملك عبدالعزبز برابغ،

المدلكة العربية السعودية

السستخلص. تخطيط القلب (تخطيط القلب الكهربائي) هو اختبار لقياس النشاط الكهربائي للقلب من خلال استخدام نبضات كهربائية.

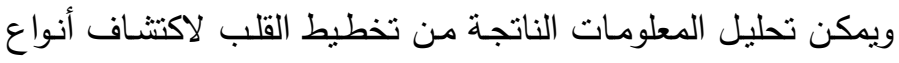
مختلفة من أمراض القلب. وهي مفيدة لمراقبة استجابة المريض لعلاج

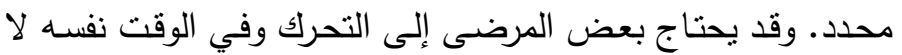

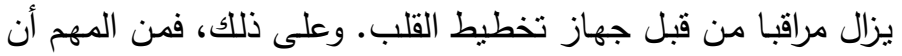
يكون هناك طريقة تتاسب تخطيط القلب المحمول. إن عملية إيجاد

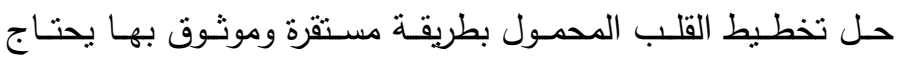

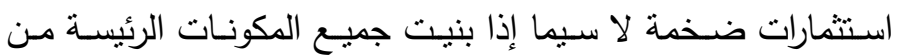

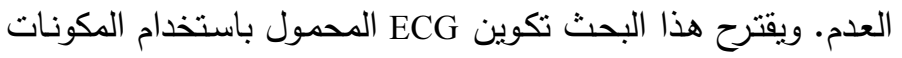
الرئيسة المتاحة على الرف. وبذلك تستخدم تقنية Bluetooth بدلا من فن فئن USB من أجل توفير قدر من المرونـة في المستقبل لإضـافة المزيد

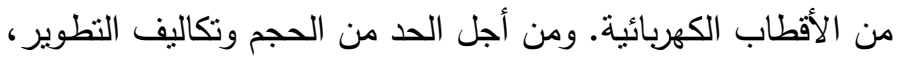
والوقت، نم استخدام الهاتف الذكي كحاسوب مضيف. ويتميز الهاتف التف الـكي الحسالي بوجـود مـوارد كافيـة لتوفير عمليـة تسـيل البيانـات اللازمة، HMI والرصد عن بعد. 